\title{
Análisis de los desafíos de las instituciones de educación superior en México
}

\author{
Diana Margarita Gutiérrez \\ dgutier@uach.mx \\ ORCID 0000-0002-0545-3442
}

Jaime Aguirre Rodríguez

jaguirrer@uach.mx

ORCID 0000-0002-1678-030X

ID de investigador de Web of Science $\underline{\text { ABC-8090-2021 }}$

Laura Georgina Carmona García

1carmona@uach.mx

ORCID 0000-0003-0314-7895

Blanca Margarita Rosas García

mrosas@uach.mx

Universidad Autónoma de Chihuahua Chihuahua, Chih., México

\section{RESUMEN}

El objetivo de esta investigación fue analizar los desafíos de la Educación Superior en México, con la finalidad de que el presente documento promueva que los estudiantes se transformen en agentes de cambio y desarrollo para la sociedad. El enfoque de esta investigación fue cuantitativo, de carácter no experimental, de tipo documental y de campo. La población de interés fueron las Universidades Públicas en México. Se aplicó un instrumento de medición que fue una encuesta a 79 universidades de la República Mexicana por medio de la Plataforma Nacional de Transparencia. Llegando a determinar con dichos estudios que, dentro de los principales resultados se encontró que el principal desafío para las Instituciones de Educación Superior, es la participación de los estudiantes en la sociedad, ya que el $54 \%$ de las universidades encuestadas respondieron estar muy de acuerdo, el $35 \%$ de acuerdo y el $8 \%$ poco de acuerdo, esto denota un interés alto en participación social. El mercado laboral exige cada vez mayor competencia y calidad de los profesionistas, la cual está ligada a la formación integral y conciencia de contribuir al desarrollo sustentable. Los egresados deben priorizar valores humanos y éticos para el beneficio personal y social.

Palabras clave: desafíos, educación, universidades, sociedad 


\title{
Analysis of the challenges facing of higher education institutions in Mexico
}

\begin{abstract}
The objective of this research was to analyze the challenges of Higher Education in Mexico, with the purpose of this document to promote students to become agents of change and development for society. The focus of this research was quantitative, nonexperimental, documentary and field research. The population of interest was public universities in Mexico. A measurement instrument was applied, which was a survey to 79 universities in Mexico through the National Transparency Platform. The main results of these studies showed that the main challenge for Higher Education Institutions is the participation of students in society, since $54 \%$ of the universities surveyed responded that they strongly agreed, $35 \%$ agreed and $8 \%$ disagreed slightly, which shows a high interest in social participation. The labor market increasingly demands greater competence and quality of professionals, which is linked to comprehensive training and awareness of contributing to sustainable development. Graduates must prioritize human and ethical values for personal and social benefit.
\end{abstract}

Keywords: challenges, education, universities, society

Artículo recibido: 02 enero 2022 Aceptado para publicación: 28 enero 2022

Correspondencia: dgutier@uach.mx Conflictos de Interés: Ninguna que declarar 


\section{INTRODUCCIÓN}

La educación superior pública con el paso del tiempo ha tenido etapas de transformación, en las cuáles ha podido reestructurar sus formas de organización y planeación para prestar atención a las necesidades de la sociedad Zamudio \& Figueroa, (2020). La sociedad está en constante cambio, lo que provoca diferentes desafíos para las Instituciones de Educación Superior (IES). Las nuevas generaciones que ingresan a la etapa universitaria tienen formas de pensar y actuar distintos, es por ello que las instituciones deben actulizarse.

La educación superior en México, se considera una educación integral por sus áreas formativas que son tres: la científica, la tecnológica y la humanística, a través de las cuales las experiencias y los conocimientos determinados por la sociedad se transmiten en los planes curriculares, el lograr un equilibrio entre estas áreas es uno de los principales objetivos en las IES (ANUIES,2009).

El objetivo de este estudio es analizar los principales desafíos de la Educación Superior en México para determinar si se está actuando para enfrentarlos.

Algunos desafíos del presente son: la participación de los estudiantes en la sociedad ante la problemática de su entorno geográfico, ofrecer cobertura con equidad, mejorar la calidad y la competencia de los profesionistas, la innovación y la formación integral de los estudiantes.

Los objetivos específicos son definir si las IES están prestando atención a los problemas de su comunidad, como lo es deterioro el medio ambiente, si permiten la apertura sobre la discusión abierta sobre los temas que afectan a la sociedad y si tienen participación en la misma.

Las instituciones de educación superior deben trabajar con sus investigadores, maestros $\mathrm{y}$ personal administrativo en formar egresados que se realicen de manera personal y profesional enfocados en el bien común, empezando por su entorno, que impacten a nivel ciudad, estado, país e incluso alcanzar el ámbito internacional.

La universidad contribuye a que una sociedad sea justa, gobernable y con oportunidades para que la calidad de vida sea cada vez mejor para todas las personas sin distinción.

La vida universitaria, además de ser una base de conocimientos, es que los estudiantes deben de aprender en las aulas su profesión junto con hábitos y valores, para resolver problemas de su entorno geográfico, en base a prácticas sociales que surjan del salón de 
clase, respaldados por su universidad de la mano de sus maestros.

De acuerdo a Silva \& Montaño, (2017); concluyen que dentro de las IES, los profesionales dedicados al trabajo social, identifican de mejor manera las demandas de la sociedad para promover cambios que tengan como objetivo el desarrollo sustentable.

Las IES, tienen la tarea de capacitar a sus maestros para que al impartir sus cátedras sean creativos, para que el estudiante aprenda a aportar mediante sus conocimientos adquiridos, ideas que tengan un impacto social siempre teniendo en cuenta la ética profesional.

La misión y visión de la institución educativa debe estar enfocada en la investigación, que de ella deriven productos científicos y tecnológicos que tengan un impacto real y positivo para el desarrollo sustentable.

Dentro de los resultados más importantes a destacar se menciona que, uno de los desafíos de las IES es la participación de los estudiantes en la sociedad, en la etapa de la universidad deben forjarse ciudadanos para que sean responsables en su pensar y actuar, en los ámbitos económicos, sociales y ambientales. Que desarrollen su carrera de manera integral, con enfoque en el bien común. La educación profesional es una etapa en la que la mayoría de los estudiantes tiene toda la vida por delante, su potencial es enorme y que mejor tomar como base de la educación la responsabilidad.

"El bien ser en todas sus interacciones genera el bien hacer y éste en cadena hace posible el bienestar del ser humano hasta producir la congruencia entre el sentir, el pensar y hacer de la sociedad" (Ponce de León, 2010).

\section{ESTRATEGIAS METODOLÓGICAS O MATERIALES Y MÉTODOS}

La naturaleza de la investigación fue cuantitativa, se aplicó un instrumento de medición que fue una encuesta basada en ("Universidad: Constuye País", 2004).

El carácter de la investigación fue no experimental, ya que no se manipularon las variables de estudio. La forma fue descriptiva para conocer la opinión de las universidades sobre el tema.

Los métodos fueron de tipo documental debido a que se utilizaron las técnicas de recolección y revisión bibliográfica y análisis de contenido.

Se tomó una muestra de 79 universidades del país por medio de la Plataforma Nacional de Transparencia, con un nivel de confianza de $95 \%$ y un margen de error del 5\%, con una probabilidad de ocurrencia del $50 \%$. 


\subsection{Planteamiento del problema}

En la actualidad las IES enfrentan grandes desafíos "el mercado de trabajo requiere profesionistas expertos en los nuevos campos científicos y tecnológicos" SánchezCastañeda, (2002); así como también egresados que contribuyan a mejorar las condiciones de desigualdad social, la pobreza, la violencia, el deterioro el medio ambiente, entre otras situaciones que afectan a la sociedad.

Algunos de los desafíos más importantes son según Sánchez-Castañeda, (2002) "La vinculación de la educación superior con el sector social y productivo; la evaluación del personal académico; reestructuración y orientación de los planes de estudio y el financiamiento de las Instituciones de la Educación Superior”.

Las universidades se enfrentan a constantes cambios, es muy importante que estén atentas a ellos, para estar en condiciones de afrontarlos, deben reorganizarse para superar los desafíos, los cuales beneficiarán a los miembros que las forman y a la sociedad en conjunto. Las universidades tendrán trascendencia en su comunidad adoptando un modelo de participación a través de sus estudiantes.

Tras lo anteriormente expuesto, es que surge el presente tema de investigación: “Análisis de los desafíos de la Educación Superior en México" del cual se desprenden los siguientes cuestionamientos.

\subsection{Pregunta General}

- ¿ Cuál es el principal desafio que enfrenta actualmente la Educación Superior en México?

\subsubsection{Preguntas específicas}

- ¿Las Instituciones de Educación Superior tienen cuidado por el medio ambiente?

- ¿Existe apertura en las Instituciones de Educación Superior para la discusión abierta sobre temas que afectan a la sociedad?

- ¿Las Instituciones de Educación Superior tienen participación en la sociedad?

\subsection{Objetivo General}

- Analizar los principales desafíos de la Educación Superior en México.

\subsubsection{Objetivos Específicos}

- Determinar si las Instituciones de Educación Superior tienen cuidado por el medio ambiente. 
- Definir si existe apertura en las Instituciones de Educación Superior para la discusión abierta sobre temas que afectan a la sociedad.

- Determinar si las Instituciones de Educación Superior tienen participación en la sociedad.

\subsection{Hipótesis General}

- La participación de los estudiantes en la sociedad es el principal desafío de la Educación Superior en México.

\subsubsection{Hipótesis específicas}

- Más del $50 \%$ de las Instituciones de Educación Superior tienen cuidado por el medio ambiente.

- En las Instituciones de Educación Superior si existe apertura para la discusión abierta sobre temas que afectan a la sociedad.

- Las Instituciones de Educación Superior si tienen participación en la sociedad.

\section{DESARROLLO}

\subsection{Marco teórico}

En la actualidad, existe consenso acerca de que los países que tendrán más posibilidades de desarrollarse en el siglo XXI serán aquellos que apuesten por ventajas comparativas basadas en la educación y calificación de su fuerza de trabajo (Lomelí, 2019).

La educación se considera como uno de los principales impulsores del desarrollo económico de los países, por lo que la inversión en ésta es base para lograr una educación de calidad que asegura ese beneficio económico en el país, Patrinos, H, (2016) de ahí la importancia en establecer su prioridad.

De acuerdo a palabras del Secretario General de la Organización para la Cooperación y Desarrollo Económico (OCDE) Gurría, (2020). La educación superior es uno de los motores del crecimiento inclusivo. Las inversiones, el acceso y la calidad de la educación superior se traducen en beneficios directos para nuestras sociedades, nuestras economías y nuestros países. Especialmente en esta era de la economía del conocimiento, que es tan competitiva y tan compleja.

Asimismo, menciona que los sistemas de educación superior accesibles y de buena calidad traen enormes beneficios a nuestras naciones: entre otras cosas, aumentan la conciencia social, fortalecen la participación democrática, aumentan los ingresos 
fiscales, reducen el gasto en transferencias sociales, reducen la desigualdad, la informalidad, la criminalidad y elevan los niveles de innovación y productividad.

Es un área en la que México tiene mucho trabajo. El 82\% de los mexicanos entre 25 y 64 años no tiene educación superior (en comparación con un promedio de la OCDE de $63 \%)$.

De acuerdo a Cruz \& Cruz, (2008) concluyen que la colaboración de las IES en los debates políticos y sociales del país fue una constante y tendría que mantenerse en el lapso del tiempo. Es fundamental que los gobiernos valoren esta contribución y ayuden al papel de las IES en el territorio, a través de la toma de decisiones y el desarrollo de políticas que den solución a los grandes desafíos del sistema ES.

De igual forma, Rodríguez, De León, \& Galarza, (2015) mencionan que las instituciones de enseñanza presentan desafíos como son la generación de conocimientos, formación de expertos integrales, competentes y con valores, oferta de un posgrado más diversificado y vinculado a la indagación, fortalecimiento del proceso de internacionalización, desarrollo de las tecnologías de la información y la comunicación y del sistemas de evaluación y colaboración de la sociedad universitaria en la administración institucional.

De acuerdo a Cuevas, (2016) menciona que "La formación y la práctica profesional sustentadas en valores para un desempeño ético, responsable y socialmente comprometido, es un desafío que implica superar el nivel puramente racional del deber ser". Las universidades deben entregar a la sociedad profesionistas formados en valores como son: respeto, responsabilidad, igualdad, dignidad, libertad, entre otros, para que su actuar sea de participación, solidaridad y bien común.

Gómez, (2011) señala que "constituye un gran reto continuar trabajando por la equidad educativa, que las instituciones se responsabilicen de los estudiantes que ingresan y ofrezcan las condiciones necesarias para que puedan desempeñarse satisfactoriamente". Dar valor a todas las personas sin distinción de razas, nacionalidades, cultura, etc., ofreciendo a cada una de ellas las mismas oportunidades durante su formación dentro de la institución.

Por otro lado Fernández, (2017) puntualiza que "La vinculación de los actores educativos con los sectores privado, social y público, así como con los entornos locales, nacionales e internacionales, se entiende como una necesidad fundamental para 
contribuir a la atención de necesidades económicas y sociales del país". La extensión universitaria es prioritaria tanto para los estudiantes, docentes e investigadores como para los entes externos ya que ambos se complementan y se benefician.

Además Fernández, (2017) propone "Establecer redes sustentadas en la perspectiva de la triple hélice que propicien la formación de Consorcios Cooperativos para la Investigación y la Innovación". La unión de esfuerzos entre la universidad, la industria y el gobierno, da como resultado profesionistas capacitados en todos los ámbitos que el entorno requiera.

García \& García, (2018) opinan que "Actuar con responsabilidad es la clave de la sostenibilidad: pensar que las generaciones actuales, como habitantes del planeta, tienen un compromiso con las nuevas generaciones, las cuales evidentemente necesitarán de los recursos que proporciona la naturaleza". Ejercer el valor de la responsabilidad permitirá a las nuevas generaciones disfrutar de la biodiversidad existente en su región.

Asimismo, García \& García, (2018) señalan que "La perspectiva de la calidad de vida se enfoca en la acumulación de bienes, se piensa que más es mejor, lo que provoca un consumismo sin importar las repercusiones a nivel personal, social y ambiental". Debe de haber más conciencia social en cuanto a los bienes materiales y de consumo para no afectar al ecosistema.

Vallaeys, De La Cruz, \& Sasia, (2009) refieren que "La Universidad tiene un impacto sobre la sociedad y su desarrollo económico, social y político”. Gran parte del futuro de las generaciones y nuestro mundo está en manos de las universidades, de su capacidad de formar personas responsables y ellas mismas ser ejemplo a seguir.

Una de las formas de acreditar que la Educación Superior tiene criterios de calidad es a través de los rankings, según establecen Van Vught, Westerhejden y Ziegele Vought, (2012) que estos son estipulados según los modelos de educación y las misiones que cumplen en la sociedad y por otra parte intereses particulares de los sujetos involucrados como docentes, estudiantes, académicos, empresarios entre otros.

De tal forma que Vallaeys, De La Cruz, \& Sasia, (2009) afirman que "la orientación curricular general, y parte de los cursos de cada carrera, tengan una relación estrecha con los problemas reales de desarrollo y puedan dictarse in situ con actores externos, bajo el método del Aprendizaje Basado en Proyectos Sociales”. Los Alumnos con asesoría de sus maestros deben poner en práctica los conocimientos adquiridos en sus 
cátedras diseñando programas para la solución de situaciones reales que se presenten en la sociedad, esto es hacer extensivo el salón de clase.

En México, la educación superior se debe alinear mejor con las necesidades cambiantes de la economía. Casi la mitad de los empleadores alertan de una falta de competencias en su sector y consideran que la educación y formación de los solicitantes de empleo no es adecuada para sus necesidades. Mejorar la relevancia y los resultados de la educación superior requiere de una visión estratégica de la educación superior, un enfoque común para todo el gobierno y la implicación de todo el sistema de educación superior. Cabe mencionar que se han hecho avances prometedores en esta dirección por medio del Comité Nacional de Productividad interministerial y el reciente marco nacional de competencias (OECD, 2019).

La agenda 2030 está basada en 17 objetivos de desarrollo sostenible, para eliminar la pobreza, la protección al planeta y mejorar las vidas y perspectivas de todas las personas, El cuarto objetivo es "Garantizar una educación inclusiva, equitativa y de calidad y promover oportunidad de aprendizaje durante toda la vida para todos" (Kliksberg, 2017).

\section{Algunas de las metas para el 2030 de este objetivo son:}

- Que todos los hombres y mujeres accedan en condiciones de igualdad a la enseñanza universitaria, otra meta es que aumente en gran medida el número de jóvenes y adultos con conocimientos técnicos y profesionales a trabajo decente y al emprendimiento (García \& García, 2018).

- Que todos los estudiantes reciban educación sobre el desarrollo sostenible, derechos humanos, igualdad de género, promoción de la paz, ciudadanía y valorización de la diversidad cultural.

- Que aumente en gran medida el número de docentes calificados con la cooperación de los países desarrollados para su formación.

México está comprometido en el cumplimiento de la Agenda 2030 para la sustentabilidad, han sido un sin número de gestiones y acciones llevadas a cabo para lograr esta meta establecida, es necesario que se gestionen políticas públicas, Carmona \& Aguirre, (2021) en este sentido y la educación superior tiene una parte fundamental, ya que la educación sustentable será la base para lograr cumplir con los objetivos de desarrollo establecidos para el 2030. 


\section{RESULTADOS Y DISCUSIÓN}

Los resultados de la encuesta fueron los siguientes, los cuales se presentan analizados.

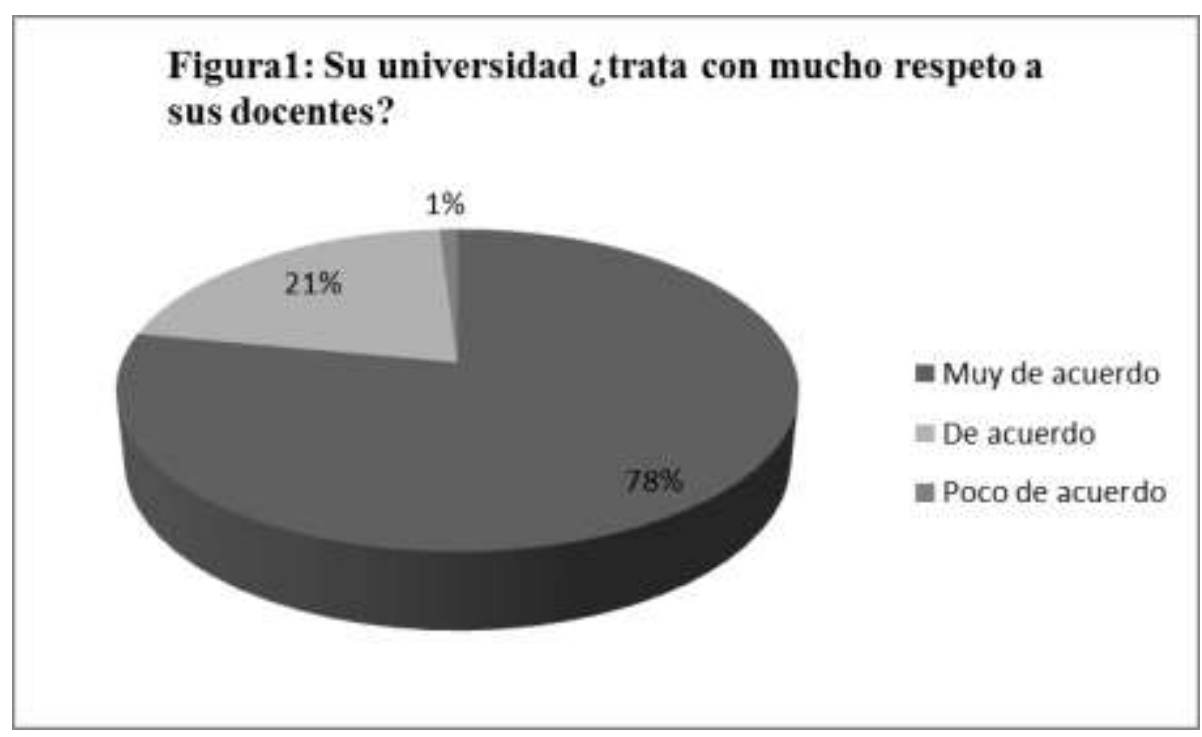

Fuente: Elaborado por los autores

De acuerdo a los resultados obtenidos el $78 \%$ de las universidades afirma estar muy de acuerdo en que trata con mucho respeto a sus docentes, el $21 \%$ respondió estar de acuerdo y solo el $1 \%$ comentan estar poco de acuerdo. Esto es un signo positivo en cualquier ámbito, el respeto es la base para el bienestar social.

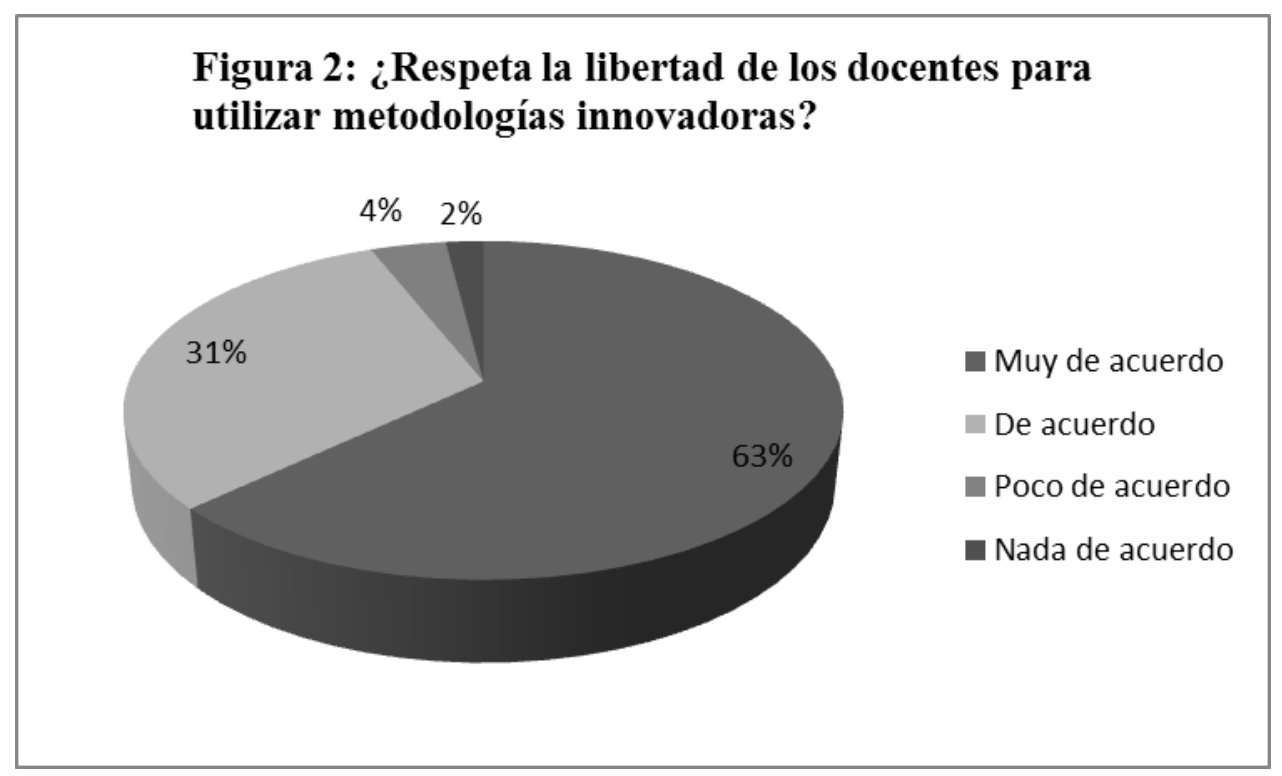

Fuente: Elaborado por los autores

Se puede observar mediante este gráfico que, si existe un gran respeto hacia la libertad de los docentes para utilizar metodologías innovadoras para la enseñanza, ya que el $63 \%$ 
respondió estar muy de acuerdo, el 31\% estuvo de acuerdo, y solo el 2\% respondió estar nada de acuerdo, esto se debe de ver reflejado en beneficio del estudiante.

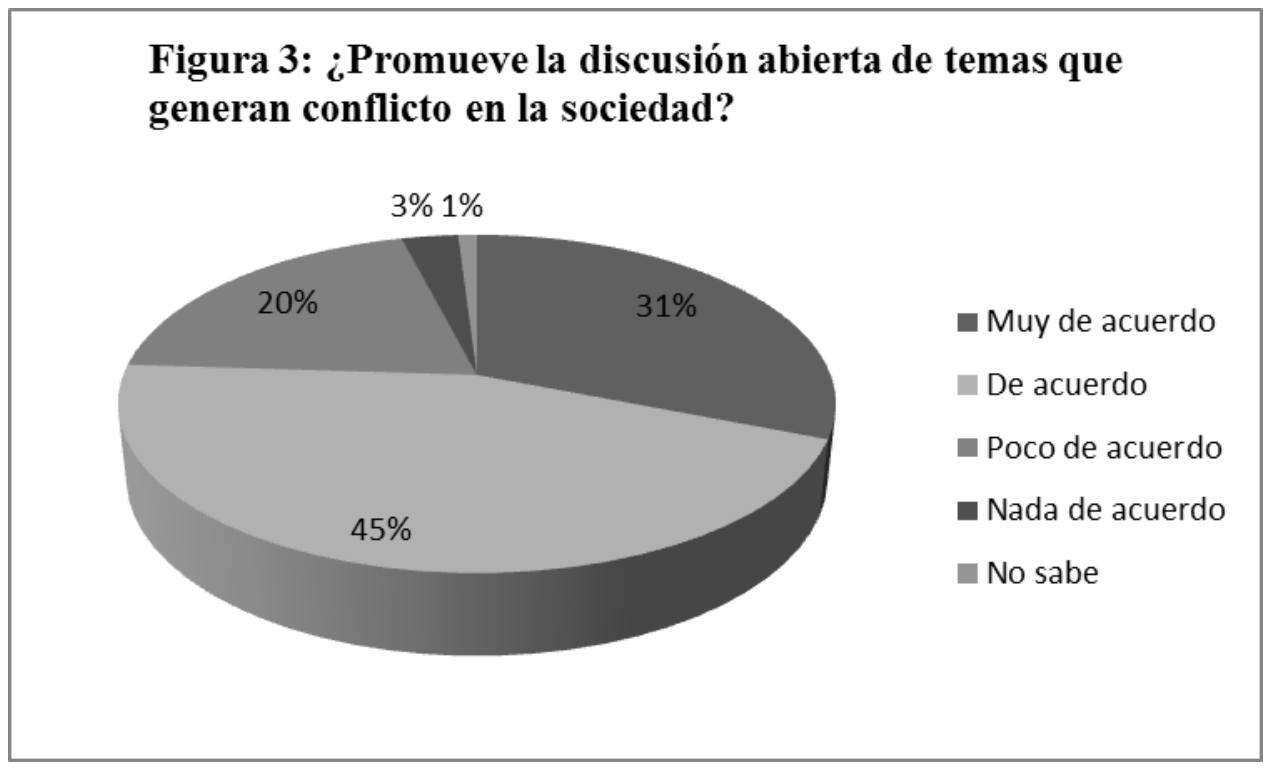

Fuente: Elaborado por los autores

La Figura 3, muestra que es alto el rango positivo de las universidades en este punto ya que el $45 \%$ de las universidades encuestadas respondieron estar muy de acuerdo en promover la discusión abierta de temas que generan conflicto en la sociedad.

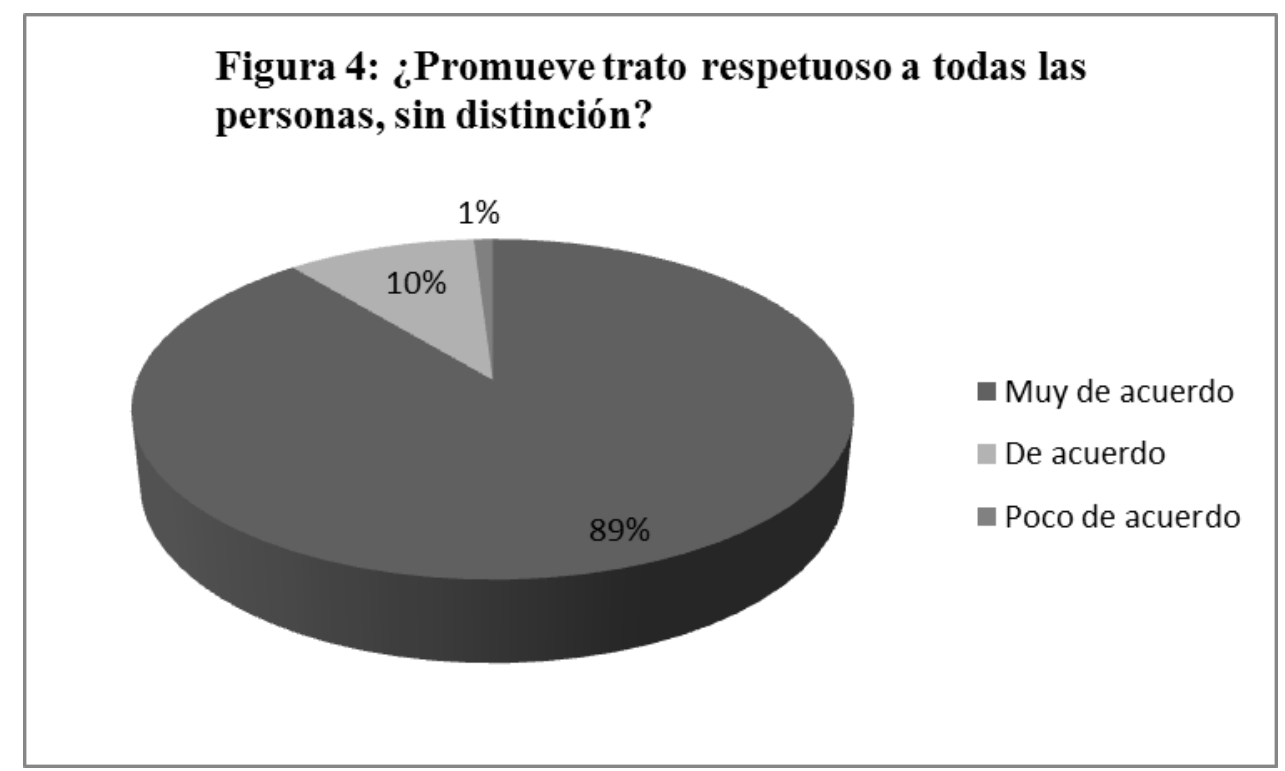

Fuente: Elaborado por los autores

Se muestra en la Figura 4, que el promover el trato respetuoso a todas las personas, sin distinción, las Instituciones de Educación Superior respondieron que el 89\% está muy de acuerdo, el $10 \%$ de acuerdo y solo el $1 \%$ poco de acuerdo. Es gratificante el rango de trato respetuoso a todas las personas. 


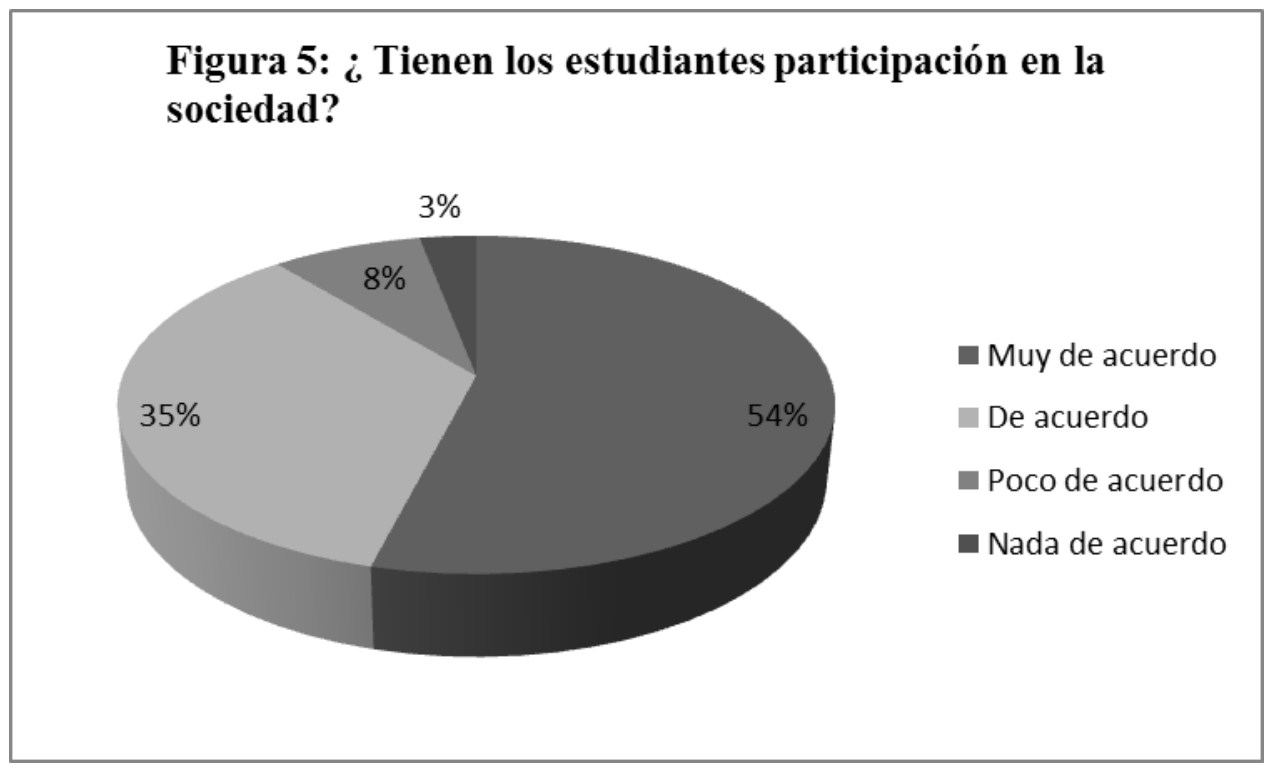

Fuente: Elaborado por los autores

La Figura 5, muestra que respecto a la participación de los estudiantes en la sociedad indica que, el $54 \%$ está muy de acuerdo, el $35 \%$ de acuerdo y el $8 \%$ poco de acuerdos, esto denota un interés alto en participación social.

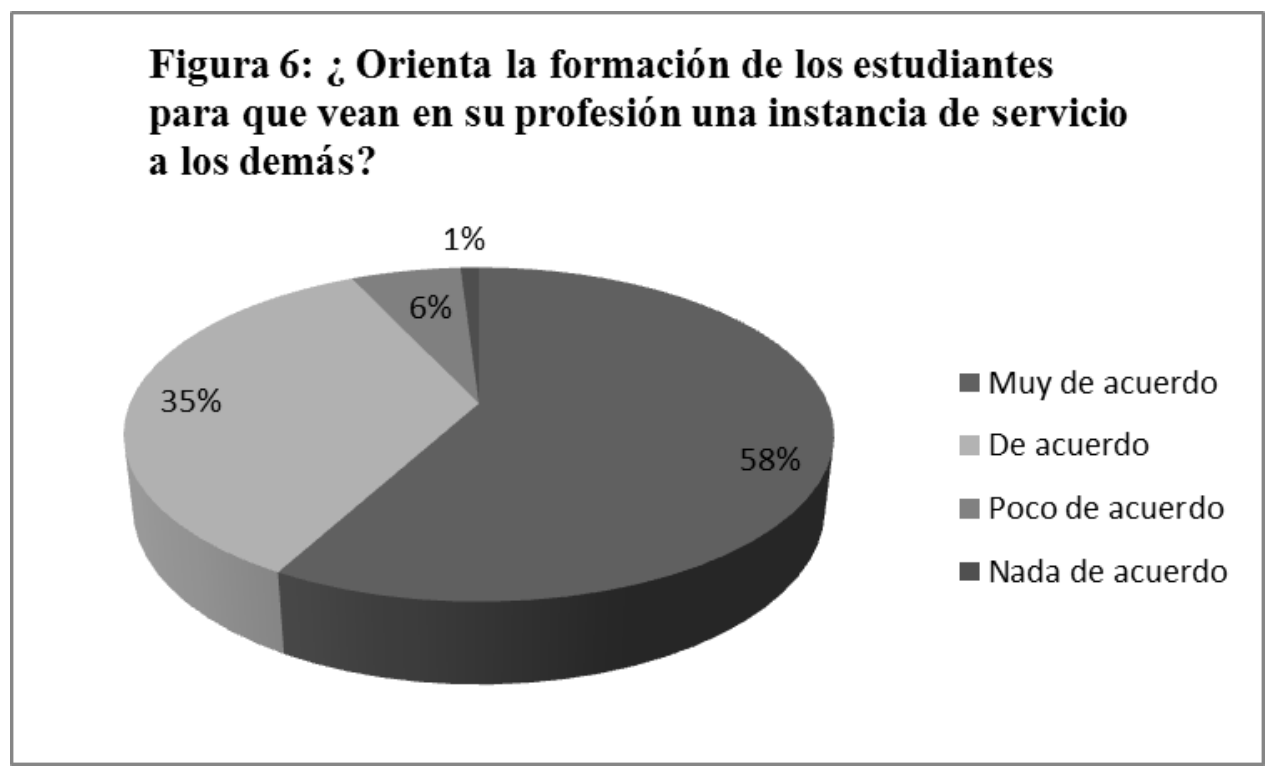

Fuente: Elaborado por los autores

Según los resultados que arroja la Figura 6 en respuesta a la pregunta sobre la orientación a la formación de los estudiantes para que vean en su profesión una instancia de servicio a los demás, el 58\% contestó estar muy de acuerdo y un $35 \%$ de 
acuerdo, lo cual nos muestra un alto sentido de solidaridad, los estudiantes se forjarán en un ambiente de responsabilidad hacia la sociedad en el ejercicio de su profesión.

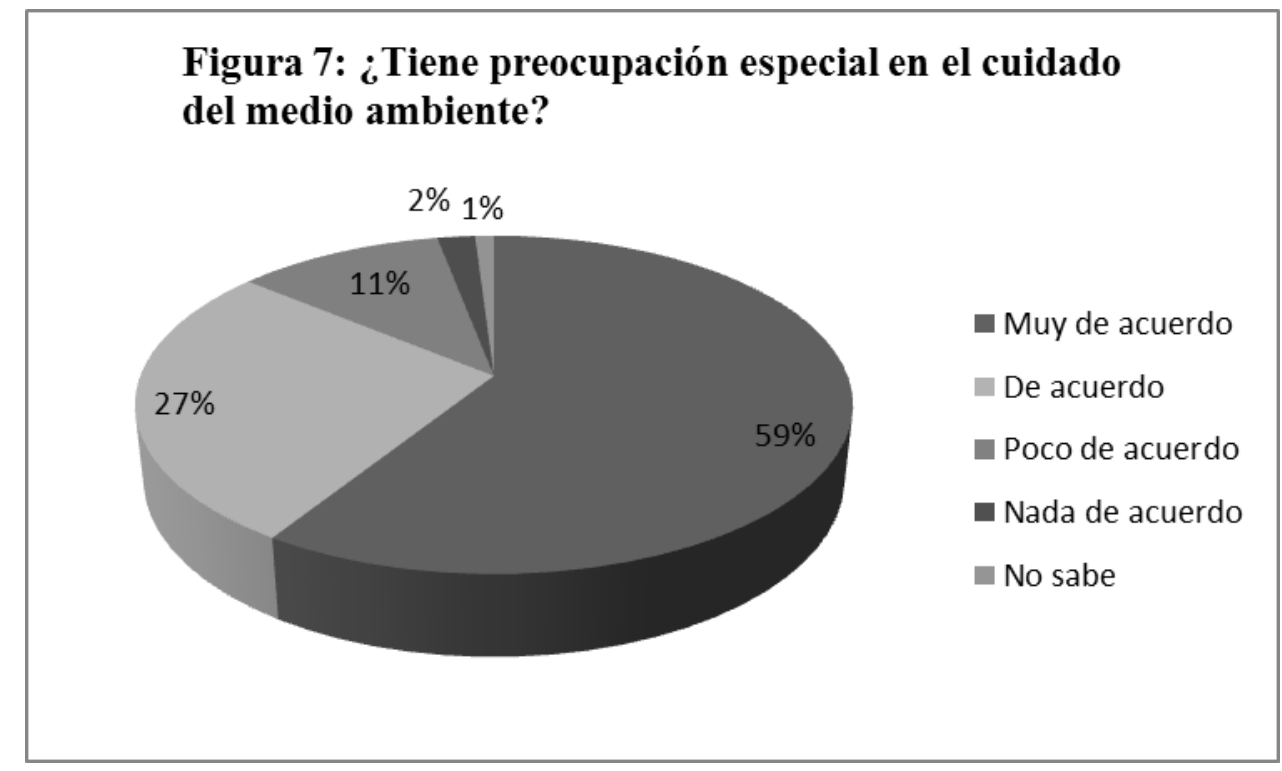

Fuente: Elaborado por los autores

La Figura 7, muestra la respuesta a la preocupación en el cuidado del medio ambiente con un $59 \%$ muy de acuerdo, $27 \%$ de acuerdo, $11 \%$ poco de acuerdo, se denota un rango aceptable de cuidado al medio ambiente.

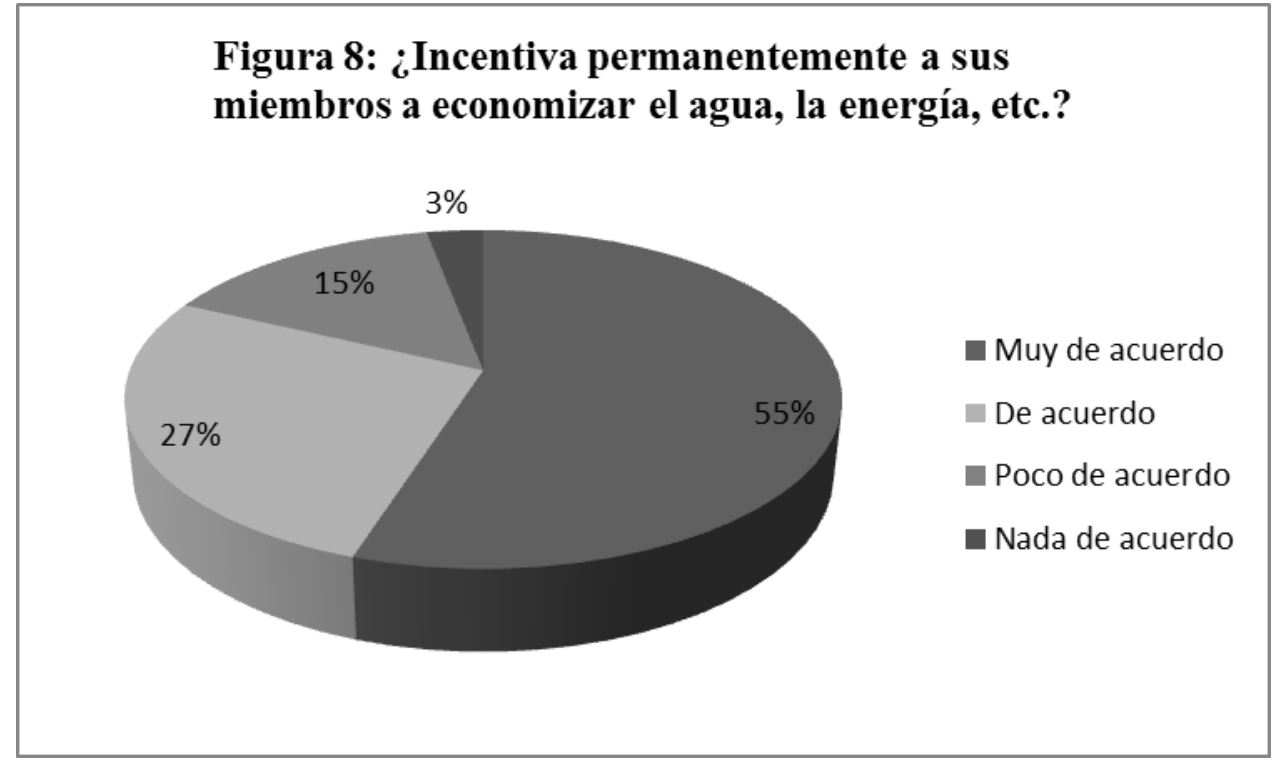

Fuente: Elaborado por los autores

Según los resultados obtenidos a la pregunta de incentivar el cuidado del agua, la energía, etc. el $55 \%$ están muy de acuerdo, el $27 \%$ de acuerdo, el $15 \%$ poco de acuerdo, la universidad debe velar para que no se destruya la diversidad ecológica. 


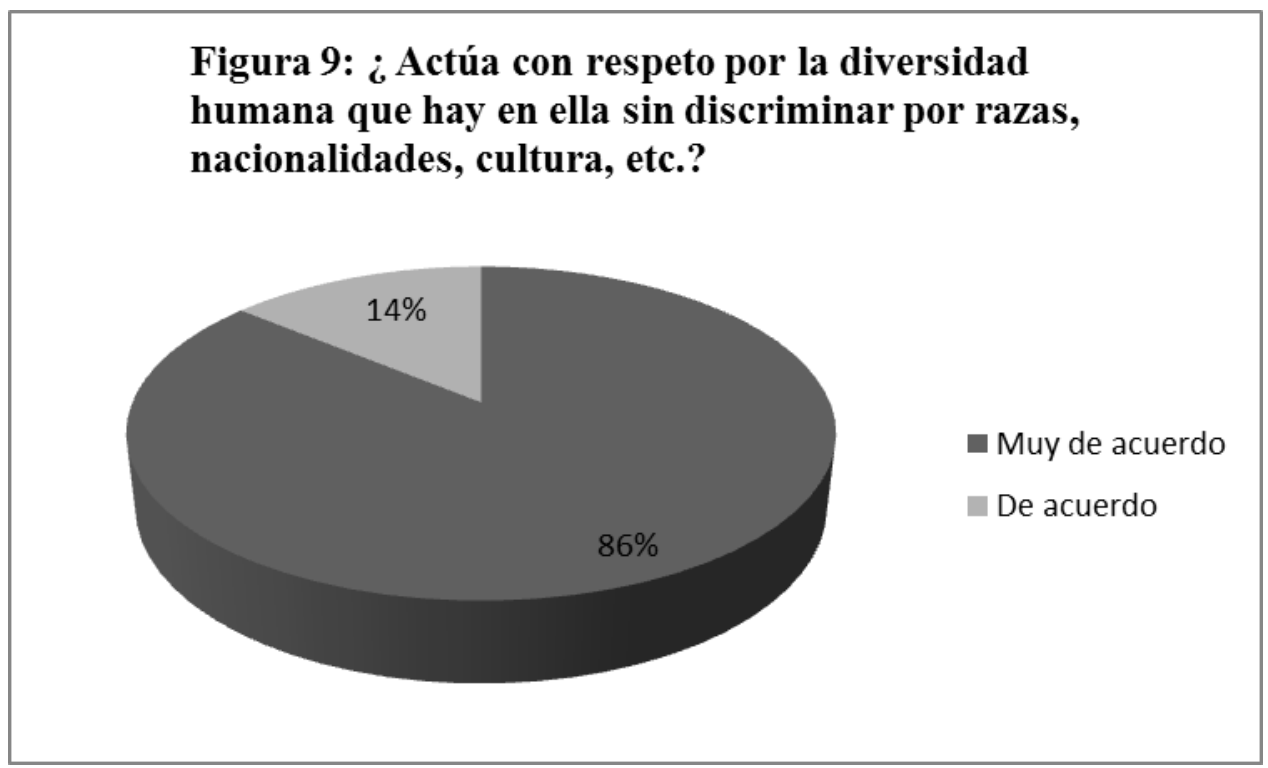

Fuente: Elaborado por los autores

La presente Figura, muestra que el $86 \%$ está muy de acuerdo en el respeto a la diversidad humana y el $14 \%$ de acuerdo, lo que demuestra la valoración de la persona y su integración a la sociedad.

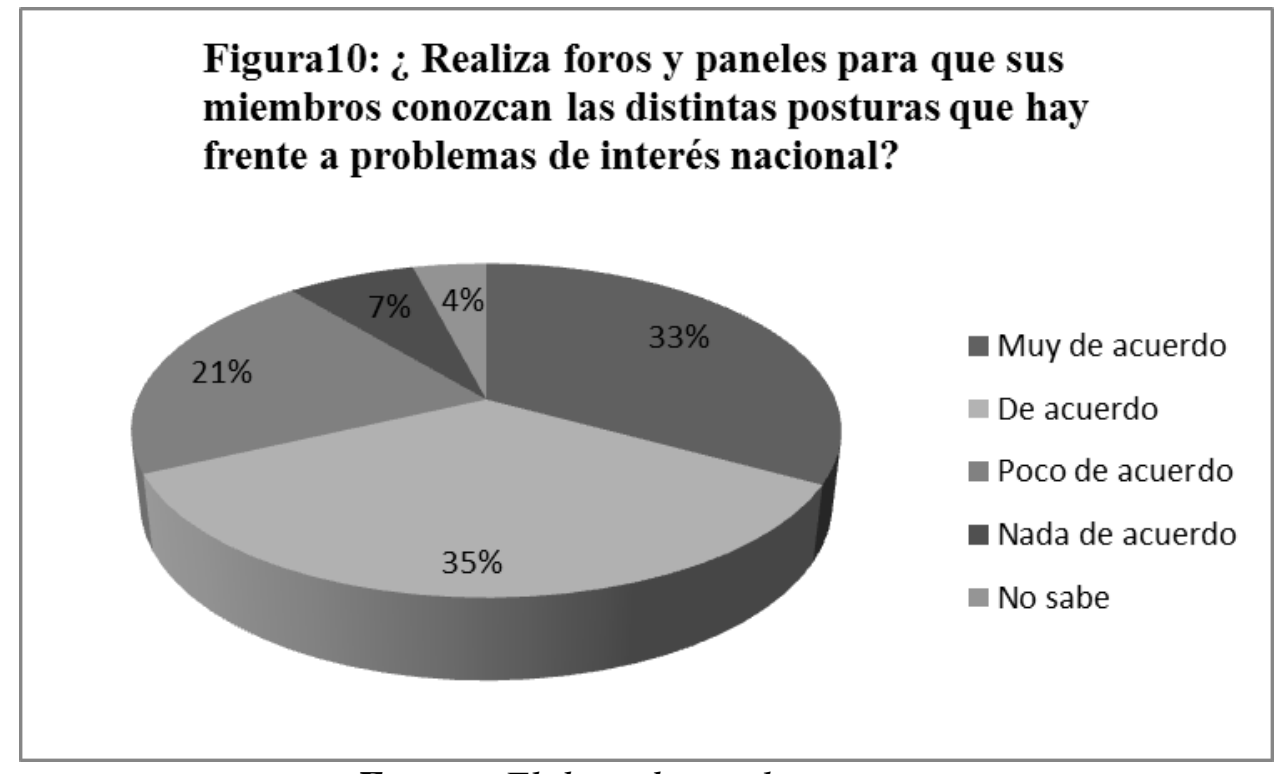

Fuente: Elaborado por los autores

En la Figura 10, se aprecia que el $33 \%$ de las universidades afirma estar muy de acuerdo que se realizan foros y paneles para que sus miembros conozcan las distintas posturas frente a problemas de interés nacional, el $35 \%$ de acuerdo, el $21 \%$ poco de 
acuerdo, el $7 \%$ nada de acuerdo, existe en las universidades un bajo rango de

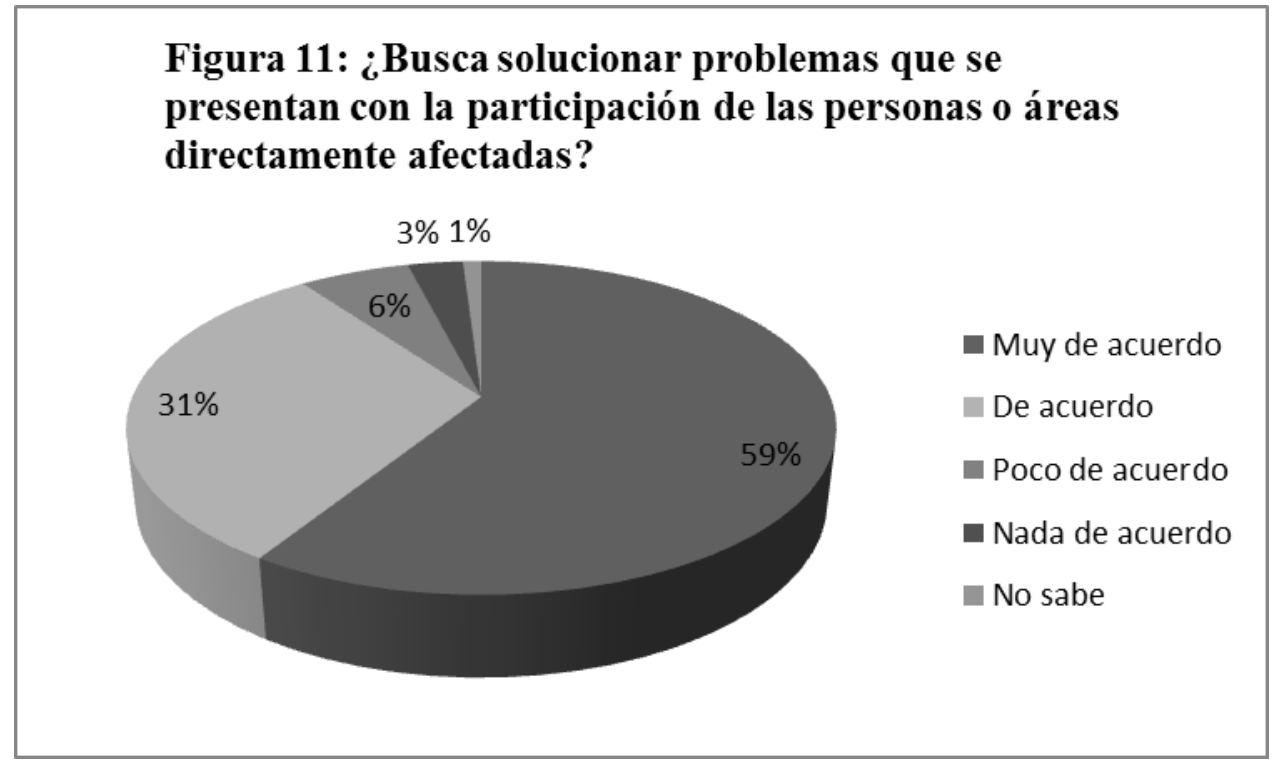

información en este punto.

Fuente: Elaborado por los autores

Se aprecia en la presente Figura que el $59 \%$ está muy de acuerdo que su Universidad busca solucionar problemas con participación de las personas o áreas afectadas, el $31 \%$ de acuerdo, el $6 \%$ poco de acuerdo, es un rango aceptable, debe existir un diálogo

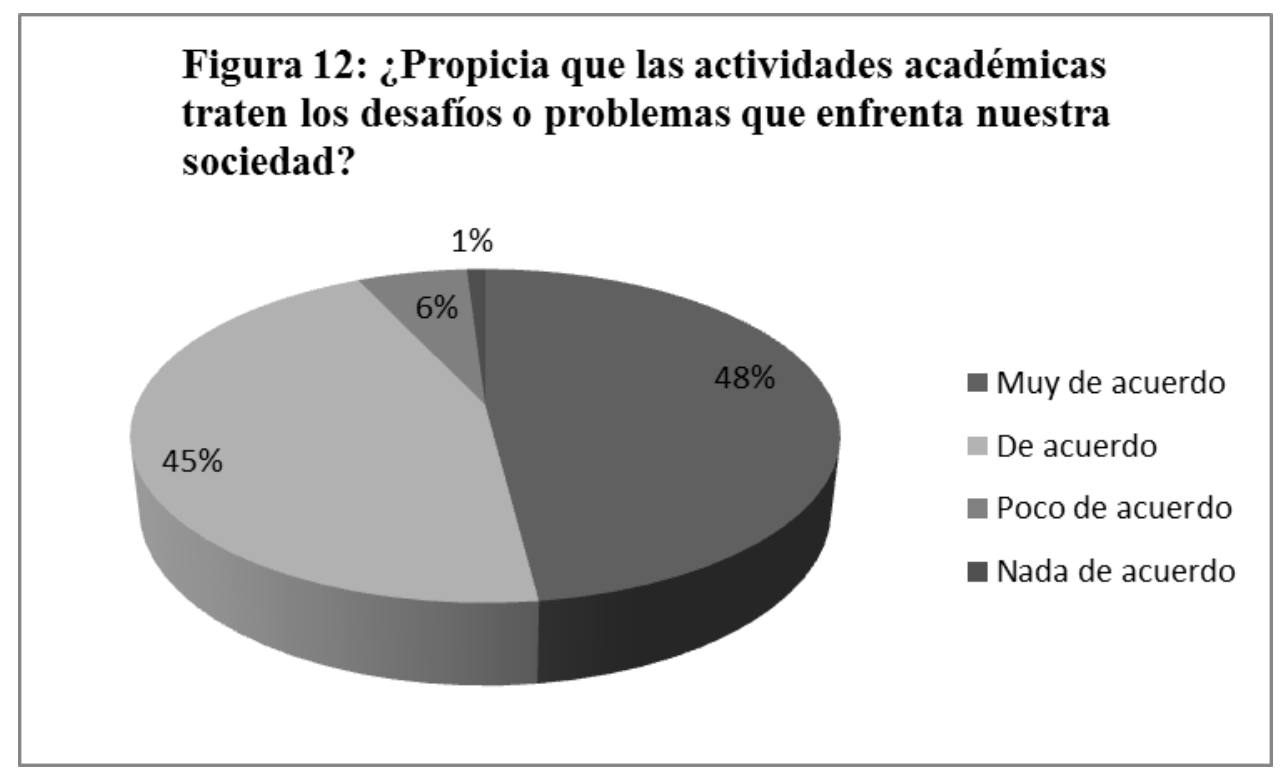

permanente entre la universidad y la sociedad.

Fuente: Elaborado por los autores 
La Figura 12, muestra que el $48 \%$ está muy de acuerdo que la Universidad propicia que las actividades académicas traten los desafíos que enfrentan la sociedad, el $45 \%$ de acuerdo, el $6 \%$ poco de acuerdo, la universidad debe entregar conocimiento a la

\section{Figura 13: ¿Promueve el vinculo de las actividades universitarias con la comunidad local?}

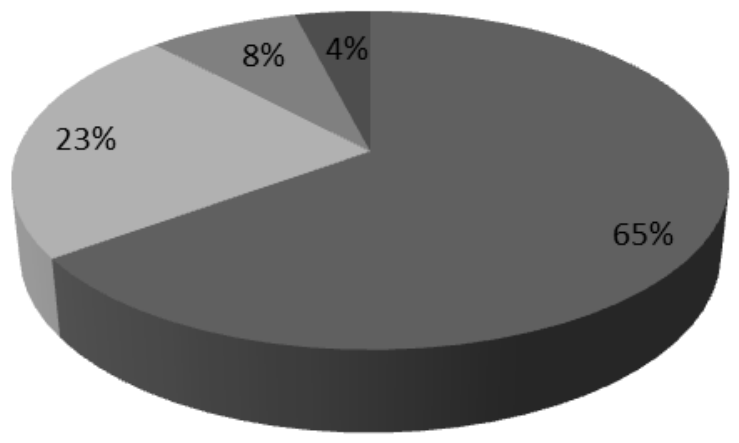

Muy de acuerdo

De acuerdo

Poco de acuerdo

- Nada de acuerdo

sociedad para la solución de problemas.

Fuente: Elaborado por los autores

Según los resultados de la Figura 13, el $65 \%$ están muy de acuerdo en promover el vínculo entre la universidad con su comunidad, el $23 \%$ de acuerdo, el $8 \%$ poco de acuerdo, se deben generar espacios para trabajar en conjunto.

\section{CONCLUSIONES}

De acuerdo a los resultados obtenidos basados en el cuestionario aplicado y a la investigación de diferentes autores, se puede concluir que la participación de los estudiantes en la sociedad es el principal desafío de las Instituciones de Educación Superior en México. Dada la información anterior, se concluye aceptada la hipótesis general, pues de acuerdo a lo que se muestra en la figura 5, indica que el $54 \%$ está muy de acuerdo y el $35 \%$ de acuerdo, esto denota un interés alto por parte de los estudiantes en la participación social.

En la figura 7 se acepta la primera hipótesis específica: más del $50 \%$ de las Instituciones de Educación Superior tienen cuidado por el medio ambiente, porque se tiene preocupación especial en este tema, con un rango del $59 \%$ muy de acuerdo y el 27 
$\%$ de acuerdo, incentivan a sus miembros a economizar el agua, la energía, etc. lo cual es un espejo para la sociedad.

En la figura 3 se acepta la segunda hipótesis específica: en las Instituciones de Educación Superior si existe apertura para la discusión abierta sobre temas que afectan a la sociedad, nos muestra que un $31 \%$ está muy de acuerdo y el $45 \%$ de acuerdo. Esto permite la reflexión sobre los problemas y la acción para resolverlos, se está trabajando para promover el debate y la participación de todos los estatus del ámbito universitario, logrando con ello beneficios para el entorno geográfico.

Respecto a la figura 4 con respecto a la promoción del trato respetuoso a todas las personas sin distinción, alcanzó un porcentaje del $89 \%$ muy de acuerdo, lo que deriva en un interés de los estudiantes por la población más vulnerable. Actúa con un alto porcentaje con respeto por la diversidad humana que hay en ella sin discriminar por razas, nacionalidades, cultura, etc.

En el resultado obtenido en la figura 12, es aceptada la segunda hipótesis específica: En las Instituciones de Educación Superior si existe apertura para la discusión abierta sobre temas que afectan a la sociedad, con un $48 \%$ muy de acuerdo y un $45 \%$ de acuerdo, en que propicia que las actividades académicas traten los desafíos o problemas que enfrenta nuestra sociedad, los egresados obtienen formación para ejercer su profesión al servicio de los demás, la universidad entrega conocimiento para la solución de los problemas de la sociedad.

Con la figura 11 se acepta la tercera hipótesis específica: Las Instituciones de Educación Superior si tienen participación en la sociedad, con un $59 \%$ muy de acuerdo y un $31 \%$ de acuerdo, las universidades buscan solucionar problemas que se presentan con la participación de las personas o áreas directamente afectadas.

El objetivo general de esta investigación fue analizar los principales desafíos de la Educación Superior en México, el cual se cumplió satisfactoriamente, porque debido a la información documentada, el resultado nos permitió revisar y comprobar un amplio panorama en el que los estudiantes universitarios con el respaldo de sus universidades actúan con y para el beneficio del entorno social y con esto se impacta a todos los niveles, comunidad, ciudad, estado y país, incluso internacionalmente.

\section{CONSIDERACIONES FINALES}


Con la experiencia obtenida en esta investigación y una vez analizados todos los datos obtenidos del cuestionario aplicado, se proponen las siguientes recomendaciones:

- El mercado laboral exige cada vez mayor competencia y calidad de los profesionistas, la cual está ligada a la formación integral y conciencia de contribuir al desarrollo sustentable. Los egresados deben priorizar valores humanos y éticos para el beneficio personal y social.

- Se requiere estar en constante actualización e innovación, es necesaria la superación continua de los docentes, la evaluación y actualización de los planes de estudio (Alonzo, s/f).

- Es recomendable realizar más foros y paneles para que sus miembros conozcan las distintas posturas que hay frente a los problemas de interés nacional.

- La mayor parte de las IES tiene un gran respeto por sus docentes y les permite utilizar metodologías innovadoras de enseñanza, esto conlleva forjar profesionistas con valores y conocimientos, capaces de ejercer autonomía, razón y conciencia, que utilicen estos elementos para cambiar la sociedad, el país y el mundo, porque para alcanzar el bienestar social solo se empieza por el respeto.

- Se recomienda que la mayoría de los docentes funjan también como investigadores para con ello crear conocimiento y a la vez enriquezcan su cátedra.

- Promover el emprendimiento, otorgarle apoyo y seguimiento, beneficia al egresado y produce un efecto dominó en la economía.

- Respecto al servicio social, requisito que debe de cumplir el estudiantado, liberarlo con proyectos específicos concluidos y respaldados con tutores.

\section{LISTA DE REFERENCIAS:}

Alonzo, V. G. (s/f). Retos, perspectivas y líneas de desarrollo de la educación superior en México y América Latina. Coepes.

ANUIES. (2009). La Educación Superior en México. Recuperado el enero de 2022, de Publicaciones

ANUIES: http://publicaciones.anuies.mx/acervo/revsup/res029/txt1c.htm

Carmona, G. L., \& Aguirre, R. J. (2021). Análisis de los beneficios de la economía circular como política pública. En Responsabilidad social y Sostenibilidad: disrupción e innovación ante el cambio de época I (págs. 622-640). México: Universidad Anáhuac. 
Cruz, L. J., \& Cruz, L. A. (1 de Agosto de 2008). Scielo Brasil. Recuperado el Enero de 2022, de La educación superior en México tendencias y desafíos: https://www.scielo.br/j/aval/a/NDYYtjTPZwGXXXvVhKV7zTz/?lang=es

Cuevas, J. A. (2016). La educación superior ante los desafíos sociales. Alteridad, 101109.

Fernández, F. E. (2017). Una mirada a los desafíos de la educación superior en México. Innovación educativa, 17(74), 183-207.

García, G. J., \& García, R. A. (2018). Percepciones y acciones de las y los estudiantes de la Universidad Autónoma del Estado de México respecto a la educación en el desarrollo sostenible. RIDE. Rev. Iberoam. Investig. Desarro. Educ.

Gómez, G. (Enero de 2011). Avances y retos en el conocimiento sobre los estudiantes mexicanos de educación superior en la primera década del siglo XXI. Perfiles educativos, 33(spe), 91-101. , 33, 91-101.

Gurría, T. J. (2020). Los desafíos y oportunidades de la educación superior en México. OCDE.

Kliksberg, B. (2017). Responsabilidad social en un mundo turbulento. Buenos Aires, Argentina: Jusbaires.

Lomelí, V. L. (mayo de 2019). Educación superior y desarrollo: los desafíos de México. Higher education and development: Mexico Challenges, 16(47).

OECD. (2019). Resultados y relevancia para el mercado laboral. En E. S. México. México: Higher Education, OECD Publishing, París.

Patrinos, H. (2016). Porque la educación es importante para el Desarrollo Económico. Recuperado el 01 de 2022, de Banco Mundial Blogs: https://blogs.worldbank.org/es/voices/por-que-la-educacion-es-importante-parael-desarrollo-economico

Ponce de León, A. L. (2010). Modelo Trans- Universal del Derecho y del Estado. México: Porrúa.

Rodríguez, V. D., De León, L. F., \& Galarza, L. J. (mayo-agosto de 2015). Los retos actuales de las instituciones de educación superior en el área de la gestión. Universidad y Sociedad, 7(2). 
Sánchez, C. A. (2002). Los retos de la educación superior: Hacia una politica de Estado. Recuperado el 27 de Enero de 2022, de UNAM: https://archivos.juridicas.unam.mx/www/bjv/libros/1/341/19.pdf

Sánchez, C. A. (2002). Los retos de la educación superior: Hacia una politica de Estado. En S. C. Alfredo, Los retos de la educación superior: Hacia una politica de Estado (pág. 265). Cd. de México: UNAM.

Sánchez, C. A. (2002). Los retos de la educación superior: Hacia una politica de Estado. En C. A. Sánchez, Los retos de la educación superior: Hacia una politica de Estado (pág. 262). Ciudad de México: UNAM.

Sánchez-Castañeda, A. (2002). Los retos de la educación superior: Hacia una politica de Estado. En A. Sánchez-Castañeda, LOS RETOS DE LA EDUCACIÓN SUPERIOR:HACIA UNA POLÍTICA DE ESTADO (pág. 265). Cd. de México: UNAM.

Silva, B. N., \& Montaño, R. W. (2017). La educación ambiental en las instituciones de educación superior públicas acreditadas en Colombia. General José María Córdova, 135.

Universidad: Constuye País. (2004). Obtenido de https://www.pucv.cl/uuaa/site/docs/20210713/20210713130254/observandolars u____.pdf

Vallaeys, F., De La Cruz, C., \& $\quad$ Sasia, P. M. (2009). http://www.upt.edu.pe/upt/sgc/assets/ckeditor/kcfinder/upload/files/MANUALrsu \%201ROS\%20PASOS.pdf. Recuperado el 20 de Diciembre de 2021

Vought, W. y. (2012). Introduction:Towards a New Ranking approach in Higer Education and Researh. Multidimensional Ranking. The Desing and Development of U-Multirank, 1-7.

Zamudio, R. F., \& Figueroa, R. A. (2020). Génesis de la responsabilidad social y su impacto en las instituciones de educación superior de México. Espacios, 41(4), 22. 